\title{
Information Transmission and Core Convergence in Quasilinear Economies*
}

\author{
Yusuke Kamishiro ${ }^{\dagger}$ and Roberto Serrano \\ Working Paper No. 2008-05 \\ January 2008
}

\begin{abstract}
We study core convergence in interim quasilinear economies with asymmetric information, concentrating on core notions in which information is transmitted endogenously within coalitions and the incentive constraints are relevant. Specifically, we shall focus on the credible core and randomized mediated core concepts. We consider independent replicas of the basic economy: independent copies of the economy in which each individual's utility only depends on the information of the individuals who belong to the same copy. We provide an example in which core convergence does not obtain for the Dutta-Vohra credible core and for Myerson's randomized mediated core. On the other hand, we establish a positive convergence result for a refinement of Myerson's core for which information disseminates across coalitions within a given random blocking mechanism. Under some conditions, this core converges to the set of incentive compatible ex-post Walrasian allocations.

Keywords: Core Convergence, Information Transmission, Coalitional Voting Mechanisms, Mediation, Rational Expectations Equilibrium. JEL Classification: C71, C72, D51, D82.
\end{abstract}

*We thank Geoffroy de Clippel for helpful comments. Serrano acknowledges the hospitality and financial support of Universidad Carlos III in Madrid, where this project began.

${ }^{\dagger}$ Dept. of Economics, Brown University, Providence, RI 02912, U.S.A.; yusuke_kamishiro@brown. edu

† Dept. of Economics, Brown University, Providence, RI 02912, U.S.A., and IMDEASocial Sciences, Madrid, Spain; roberto_serrano@brown.edu 


\section{Introduction}

The core convergence theorem is an important cornerstone of the relationship between the predictions of game theory in large economies and competitive equilibrium allocations. Many results have followed the seminal works of Debreu and Scarf (1963) and Aumann (1964); see Anderson (2008) for a recent survey. This paper focuses on interim economies with asymmetric information, and concentrates on core concepts that model the endogenous information transmission that goes on within each coalition. In particular, three core concepts will feature prominently in the paper: the credible core found in Dutta and Vohra (2005), the virtual utility core of Myerson (2007), and the randomized mediated core of Serrano and Vohra (2007).

The core convergence question in the ex-ante stage (i.e., that in which no agent has received any private information) has also been studied. For this stage, if no restrictions are imposed on the set of allocations, the question is simply a reformulation of the standard problem in an Arrow-Debreu economy framework. Indeed, the most interesting cases happen when either measurability or incentive compatibility restrictions are imposed; the reader is referred to Forges, Minelli and Vohra (2002) for an excellent general survey of the area, and to Serrano, Vohra and Volij (2001) for a discussion. There are also results obtained for the ex-post stage (i.e., that in which all private information has been made common knowledge); see, for example, Einy, Moreno and Shitovitz (2000). ${ }^{1}$ The results in the current paper will connect with some of these, due to the information transmission embodied in the interim cores we study, which place them closer to ex-post solution concepts.

For the interim stage, the most closely related study to the current paper is the work by Serrano, Vohra and Volij (2001). That paper studies replicas in which the set of states of the world is not replicated with the economy, which implies that, already in the second replica, the economy is one of non-exclusive information in the sence of Postlewaite and Schmeidler (1986) and incentive constraints become redundant. The general message of that paper is a robust failure of the core convergence theorem in these settings (this applies to several versions of the interim core and the interim price

\footnotetext{
${ }^{1}$ These authors work with atomless economies, although they assume the number of states is finite. If we tried to extend our analysis to atomless economies, the state space would also be "atomless." Then, it would be difficult to define the joint information of a coalition, for example, hindering the use of the arguments in that paper.
} 
equilibrium). Essentially, for cores that use a limited amount of exogenously specified information transmission (e.g., the coarse core of Wilson (1978)), the core is "too large" and does not shrink down to the set of price allocations; and the core that allows unrestricted communication (Wilson's fine core) is often "too small," even empty, still when price allocations can be supported.

In the current paper, we shall consider independent replicas, those introduced in Gul and Postlewaite (1992) and also studied in Forges, Heifetz and Minelli (2001). Independent replicas also replicate the states of the world, and in them, each agent's preferences depend only on the information pertinent to his replica. We shall concentrate on economies in which the state is not verifiable ex-post, and thus, incentive constraints become important. For these economies, some recent papers have proposed core notions in which communication within coalitions is endogenous; these core notions take center stage in our study and we describe them in the sequel. ${ }^{2}$

The credible core, proposed in Dutta and Vohra (2005), is the set of incentive compatible allocations immune to credible objections. A coalition has a credible objection if it can identify an informational event such that the types of agents involved in the event are the only ones that prefer the alternative proposed to the status quo, given that the other types behave as prescribed in the objection. This self-selection ensures that a type wishes to participate in the objection if and only if it is consistent with the objection's event. Therefore, the information transmitted in the objection via the event is "credible" in that the types that are not part of it have no incentive to join the objection; hence the name of the concept. Based on the virtual utility construct, Myerson (2007) proposes a core notion that, in addition to the credibility requirements, considers random coalition formation and random allocations for each coalition. Finally, Serrano and Vohra (2007) set up the Dutta-Vohra and Myerson objections as communication mechanisms played by the agents in each coalition, and derive their objections' inequalities from the equilibria of such communication games. When random blockings are possible, one difference between Myerson's (2007) concept and that arrived at in Serrano and Vohra (2007) had not been previously emphasized, and such a difference plays an important role in our results. The issue concerns the possibility of information transmission within a random blocking plan across the different coalitions that comprise the plan. Correspondingly, we

\footnotetext{
${ }^{2}$ In the last section, we shall discuss how our results extend to economies without incentive constraints.
} 
shall talk about the credible core of Dutta-Vohra, the virtual utility core of Myerson and the randomized utility core of Serrano-Vohra. Details will be provided in Section 2 about these three nested sets of core allocations.

We shall assume that agents in our economies have quasilinear preferences. For this class, different core existence results have been provided (see Dutta and Vohra (2005), Myerson (2007)). It is important to stress that non-emptiness is in general hard to achieve for all these core concepts once incentive constraints are in place, as pointed out by Vohra (1999) and Forges, Mertens and Vohra (2002). Aside non-emptiness, as the reader will appreciate, some of our proofs also rely on the quasilinearity assumption. Myerson (2007) notes that, even in quasilinear settings, random blockings make a difference to the definition of the core in the presence of asymmetric information (unlike what is known for complete information games). The current work shows that, even in quasilinear economies, depending on how much information transmission one allows in random blocking plans, the answer that one obtains to the convergence question is also very different.

Our results show both negative and positive convergence conclusions. First, we shall propose a simple robust example to demonstrate that Myerson's (2007) virtual utility core -and thus, Dutta and Vohra's (2005) credible core- does not converge to any price equilibrium notion. However, if information transmission is allowed to flow within the coalitions that participate in a given random blocking plan, as modelled in Serrano and Vohra (2007), the equal-treatment strictly incentive compatible allocations in the randomized mediated core converge to the set of incentive compatible ex-post Walrasian allocations, whenever this is non-empty. The latter result may shed light on the rather mysterious information transmission that goes on within a price function in a fully revealing rational expectations equilibrium.

\section{Preliminaries}

\subsection{An Interim Exchange Economy}

The basic model of an exchange economy with asymmetric information that we shall use in this paper can be formulated as follows. Let $T_{i}$ denote the (finite) set of agent $i$ 's types. The interpretation is that $t_{i} \in T_{i}$ denotes the private information possessed by agent $i$. With $N=\{1, \ldots, n\}$ as the finite set of agents, let $T_{N}=\prod_{i \in N} T_{i}$ denote the set of all information states. 
We will use the notation $t_{-i}$ to denote $\left(t_{j}\right)_{j \neq i}$. Similarly $T_{-i}=\prod_{j \neq i} T_{j}$, $T_{S}=\prod_{j \in S} T_{j}$ and $T_{-S}=\prod_{j \notin S} T_{j}$. We assume that agents have a common prior probability distribution $q$ defined on $T_{N}$, and that no type is redundant, i.e., $q\left(t_{i}\right)>0$ for all $t_{i} \in T_{i}$ for all $i$. At the interim stage, nature chooses $t_{N} \in T_{N}$, and each agent $i$ knows her type, $t_{i}$. Hence, conditional probabilities will be important: for each $i \in N$ and $t_{i} \in T_{i}$, the conditional probability of $t_{-i} \in T_{-i}$, given $t_{i}$ is denoted $q\left(t_{-i} \mid t_{i}\right)$.

We assume that there are $|L|=l$, a finite number of commodities, and that commodity $L$ is a nummeraire. The consumption set of agent $i$ is $X_{i} \subseteq$ $R_{+}^{l-1} \times R$. Agent $i$ 's utility function in state $t_{N}$ is denoted $u_{i}\left(\cdot, t_{N}\right): X_{i} \times T_{N} \mapsto$ $R$, and it is quasilinear in the nummeraire: $u_{i}\left(x_{i}, t_{N}\right)=v_{i}\left(x_{i}^{-l}, t_{N}\right)+x_{i}^{l}$. The endowment of agent $i$ of type $t_{i}$ is $\omega_{i} \in X_{i}$ (assumed to be independent of the state - with this assumption, all private information concerns agents' preferences and beliefs.)

We can now define an admissible exchange economy as $\mathcal{E}=\left\langle\left(u_{i}, X_{i}, \omega_{i}\right.\right.$, $\left.\left.T_{i}\right)_{i \in N}, q\right\rangle$.

In our analysis, the contracts will be signed at the interim stage. However, when a coalition gets together to make a proposal to upset a given allocation, some information may be transmitted within the members of the coalition. We shall allow coalitions to use random incentive compatible mechanisms (e.g., Myerson (2007), Serrano and Vohra (2007)). We begin by describing deterministic allocation rules.

\subsection{Deterministic Mechanisms}

For coalition $S \subseteq N$, a feasible deterministic (state contingent) $S$-allocation, $x: T_{N} \mapsto R^{l s}$ (where $s$ denotes the cardinality of $S$ ), consists of a commodity bundle for each consumer in $S$ in each state such that $\sum_{i \in S} x_{i}\left(t_{N}\right) \leq \sum_{i \in S} \omega_{i}$ for all $t_{N} \in T_{N}$, and satisfying that $x\left(t_{S}, t_{-S}^{\prime}\right)=x\left(t_{S}, t_{-S}^{\prime \prime}\right)$ for all $t_{S} \in T_{S}$ and for all $t_{-S}^{\prime}, t_{-S}^{\prime \prime} \in T_{-S}$. (The latter assumption is made to exclude basic externalities across coalitions, i.e., the set of feasible allocations to a coalition is independent of the information held by the complement, although this may affect the utilities of agents in the coalition). We will denote by $\mathcal{A}_{S}$ the set of feasible deterministic state contingent allocations of $S$. With confusion being avoided by the context, we shall also use $\mathcal{A}_{S}$ to denote the set of feasible deterministic allocations in a given state: $\mathcal{A}_{S}=\left\{\left(x_{i}\right) \in \prod_{i \in S} X_{i} \mid \sum_{i \in S} x_{i} \leq\right.$ $\left.\sum_{i \in S} \omega_{i}\right\}$. Similarly, deterministic state contingent $N$-allocations are simply referred to as deterministic allocations, and the set of such deterministic 
allocations is denoted by $\mathcal{A}_{N}$.

For most of this paper, we shall work in environments in which the information state will not be verifiable, not even at the ex-post stage. Thus, it becomes necessary to impose the incentive compatibility constraints into the analysis.

We begin again by considering deterministic allocations. Then, if agent $i$ of type $t_{i}$ pretends to be of type $t_{i}^{\prime}$ (while all other agents are truthful), she gets interim utility:

$$
U_{i}\left(x, t_{i}^{\prime} \mid t_{i}\right)=\sum_{t_{-i} \in T_{-i}} q\left(t_{-i} \mid t_{i}\right) u_{i}\left(x_{i}\left(t_{-i}, t_{i}^{\prime}\right),\left(t_{-i}, t_{i}\right)\right) .
$$

An allocation $x$ is incentive compatible if for every $i \in N$, and for every $t_{i} \in T_{i}$

$$
U_{i}\left(x \mid t_{i}\right) \geq U_{i}\left(x, t_{i}^{\prime} \mid t_{i}\right)
$$

for every $t_{i}^{\prime} \in T_{i} \backslash\left\{t_{i}\right\}$. We denote the set of incentive compatible allocations by $\mathcal{A}_{N}^{*}$. An allocation $x$ is strictly incentive compatible if all these inequalities are strict.

Information transmission concerns ruling out some states as impossible, through the identification of smaller events. For an event $E \subseteq T_{N}$ and $t_{i} \in T_{i}$, let

$$
E_{-i}\left(t_{i}\right)=\left\{t_{-i} \in T_{-i} \mid\left(t_{i}, t_{-i}\right) \in E\right\}
$$

and

$$
E_{i}=\left\{t_{i} \in T_{i} \mid E_{-i}\left(t_{i}\right) \neq \emptyset\right\} .
$$

Consider an allocation rule $x \in \mathcal{A}_{N}$, agent $i$ of type $t_{i}$ and an event $E$. Suppose $q\left(E_{-i}\left(t_{i}\right)\right)>0$. Then the interim utility conditional on $E$ can be expressed as:

$$
U_{i}\left(x \mid t_{i}, E\right)=\sum_{t_{-i} \in E_{-i}\left(t_{i}\right)} \frac{q\left(t_{-i} \mid t_{i}\right)}{q\left(E_{-i}\left(t_{i}\right) \mid t_{i}\right)} u_{i}\left(x_{i}\left(t_{-i}, t_{i}\right),\left(t_{-i}, t_{i}\right)\right) .
$$

The corresponding interim utility (conditional on $E$ ) if type $t_{i}$ pretends to be of type $t_{i}^{\prime}$, while the others are truth-telling, is:

$$
U_{i}\left(x, t_{i}^{\prime} \mid t_{i}, E\right)=\sum_{t_{-i} \in E_{-i}\left(t_{i}\right)} \frac{q\left(t_{-i} \mid t_{i}\right)}{q\left(E_{-i}\left(t_{i}\right) \mid t_{i}\right)} u_{i}\left(x_{i}\left(t_{-i}, t_{i}^{\prime}\right),\left(t_{-i}, t_{i}\right)\right) .
$$

Given $E \subseteq T_{N}$, an $S$-allocation $x \in \mathcal{A}_{S}$ is incentive compatible over $E$ if for every $i \in S$ and for every $t_{i}, t_{i}^{\prime} \in E_{i}$ :

$$
U_{i}\left(x \mid t_{i}, E\right) \geq U_{i}\left(x, t_{i}^{\prime} \mid t_{i}, E\right) .
$$




\subsection{Random Mechanisms}

Now we expand coalitional interactions and information transmission to also consider random plans. A random coalitional plan $\mu$ consists of a probability distribution of feasible allocation mechanisms for various coalitions. In particular, $\mu\left(S, y^{S}, t_{N}\right)$, where $y^{S} \in \mathcal{A}_{S}$, denotes the probability with which coalition $S \subseteq N$ is receiving $y^{S} \in \mathcal{A}_{S}$ when the (reported) state is $t_{N} \in T_{N}$.

We shall say that such a random plan is measurable with respect to coalitional information, or simply measurable, if for every $S$, for every $y^{S}$ and for every $t_{S}, \mu\left(S, y^{S},\left(t_{S}, t_{-S}^{\prime}\right)\right)=\mu\left(S, y^{S},\left(t_{S}, t_{-S}^{\prime \prime}\right)\right)$ for every $t_{-S}^{\prime}, t_{-S}^{\prime \prime} \in T_{-S}$. Otherwise, we shall say that the random plan is non-measurable. Nonmeasurabilities in this sense are in principle possible, although we shall always require that, if $P$ is the union of all coalitions in the support of $\mu$, $\mu\left(S, y^{S},\left(t_{P}, t_{-P}^{\prime}\right)\right)=\mu\left(S, y^{S},\left(t_{P}, t_{-P}^{\prime \prime}\right)\right)$ for all $\left(S, y^{S}\right)$ in the support of $\mu$, all $t_{P} \in T_{P}$ and all $t_{-P}^{\prime}, t_{-P}^{\prime \prime} \in T_{-P}$. The idea is to think of the random plan as a mediated communication mechanism used by the members of the coalition; thus, information can potentially flow in any possible way within the mechanism, but only information available to someone that participates in the random plan.

Note that, if $\mu\left(S, y^{S}, t_{N}\right)>0$ implies $\mu\left(S, z^{S}, t_{N}\right)=0$ for all $z^{S} \neq y^{S}$, we would associate with each coalition $S$ one deterministic allocation $y^{S} \in \mathcal{A}_{S}$. But in general a random plan may include random allocations within each coalition, as well as random coalition formation. The plan specifies for each $t_{N} \in T_{N}, 0 \leq \mu\left(S, y^{S}, t_{N}\right) \leq 1$ for all coalitions $S$ and $S$-allocations $y^{S}$, and $\sum_{S} \sum_{y^{S}} \mu\left(S, y^{S}, t_{N}\right) \leq 1$.

For type $t_{i}$ of agent $i$, the interim utility of such a random plan $\mu$, completed with the implementation of the status quo $x$ as needed, is this:

$$
\begin{aligned}
U_{i}\left([\mu / x] \mid t_{i}\right)=\sum_{t_{-i}} q\left(t_{-i} \mid t_{i}\right)\left[\sum_{S \supseteq\{i\}} \sum_{y^{S}} \mu\left(S, y^{S}, t_{N}\right) u_{i}\left(y_{i}^{S}\left(t_{N}\right), t_{N}\right)\right. & \\
& \left.+\nu_{i}\left(t_{N}\right) u_{i}\left(x_{i}\left(t_{N}\right), t_{N}\right)\right],
\end{aligned}
$$

where $\nu_{i}\left(t_{N}\right):=1-\sum_{S \supseteq\{i\}} \sum_{y^{S}} \mu\left(S, y^{S}, t_{N}\right)$.

If everyone else is truthful and type $t_{i}$ pretends to be type $t_{i}^{\prime}$, his interim expected utility from the random plan $\mu$ completed with the status quo $x$ is:

$$
\begin{array}{r}
U_{i}\left([\mu / x], t_{i}^{\prime} \mid t_{i}\right)=\sum_{t_{-i}} q\left(t_{-i} \mid t_{i}\right)\left[\sum_{S \supseteq\{i\}} \sum_{y^{S}} \mu\left(S, y^{S},\left(t_{-i}, t_{i}^{\prime}\right)\right) u_{i}\left(y_{i}^{S}\left(t_{-i}, t_{i}^{\prime}\right), t_{N}\right)\right. \\
\left.+\nu_{i}\left(t_{-i}, t_{i}^{\prime}\right) u_{i}\left(x_{i}\left(t_{N}\right), t_{N}\right)\right]
\end{array}
$$


where $\nu_{i}\left(t_{i}^{\prime}, t_{-i}\right):=1-\sum_{S \supseteq\{i\}} \sum_{y^{S}} \mu\left(S, y^{S},\left(t_{i}^{\prime}, t_{-i}\right)\right)$.

Note how the type misreport affects the implementation probabilities $\mu(\cdot)$ of each coalitional allocation and $\nu_{i}(\cdot)$ of the status quo. In addition, it garbles the outcomes in each $y^{S}$, but not those in $x$. In Section 4 , we shall go over this, which is related to the timing of events in the blocking plan versus the status quo; see also Myerson (2007) and Serrano and Vohra (2007).

A random plan $\mu$ is incentive compatible if for every $i \in N$ and for every $t_{i}, t_{i}^{\prime}$ in the support of $\mu$ :

$$
U_{i}\left([\mu / x] \mid t_{i}\right) \geq U_{i}\left([\mu / x], t_{i}^{\prime} \mid t_{i}\right) .
$$

\subsection{Core Notions}

These concepts have been used to define several versions of the core in interim economies with asymmetric information. It is important to note that, when coupled with an incentive compatible allocation $x \in \mathcal{A}_{N}^{*}$, interpreted as the status quo, a random plan trying to upset it may allow for the status quo to prevail with some positive probability. That is, since a random plan $\mu$ against the presence of status quo $x$ allows for each $t_{N} \in T_{N}, 0 \leq \mu\left(S, y^{S}, t_{N}\right) \leq 1$ for all coalitions $S$ and $S$-allocations $y^{S}$, and $\sum_{S} \sum_{y^{S}} \mu\left(S, y^{S}, t_{N}\right) \leq 1$, one must assign the rest of probability $\nu\left(t_{N}\right)$ to the implementation of the status quo. ${ }^{3}$ Following Myerson (2007) and Serrano and Vohra (2007), the implementation of the status quo is not affected by the reported types in the blocking plan (we shall explain these timing issues in Section 4).

Given an incentive compatible allocation $x \in \mathcal{A}_{N}^{*}$, a random plan $\mu$ is a random blocking plan against $x$ whenever for every type $t_{i} \in T_{i}$ :

$$
U_{i}\left([\mu / x] \mid t_{i}\right) \geq U_{i}\left(x \mid t_{i}\right)
$$

with at least one strict inequality, and

$$
U_{i}\left([\mu / x] \mid t_{i}\right) \geq U_{i}\left([\mu / x], t_{i}^{\prime} \mid t_{i}\right)
$$

for all $t_{i}^{\prime} \in T_{i}$.

\footnotetext{
${ }^{3}$ The probabilities $\nu_{i}(\cdot)$ that appear in the definitions of $U_{i}([\mu / x] \mid \cdot)$, right before the current subsection, are simply the marginals of $\nu$. Also, when the appropriate payoff inequalities are imposed versus a given status quo, one describes a "blocking plan" used in the definition and characterization of the inner core; see Myerson (1991), Qin (1993) and de Clippel and Minelli (2005).
} 
Equivalently, one can normalize probabilities and simplify these inequalities because of identical terms on both sides, and write:

$$
\sum_{t_{-i}} q\left(t_{N}\right) \sum_{S \supseteq\{i\}} \sum_{y^{S}} \mu\left(S, y^{S}, t_{N}\right)\left[u_{i}\left(y_{i}^{S}\left(t_{N}\right), t_{N}\right)-u_{i}\left(x_{i}\left(t_{N}\right), t_{N}\right)\right] \geq 0
$$

for all $t_{i} \in T_{i}$, with at least one strict inequality, and

$$
\begin{gathered}
\sum_{t_{-i}} q\left(t_{N}\right) \sum_{S \supseteq\{i\}} \sum_{y^{S}} \mu\left(S, y^{S}, t_{N}\right)\left[u_{i}\left(y_{i}^{S}\left(t_{N}\right), t_{N}\right)-u_{i}\left(x_{i}\left(t_{N}\right), t_{N}\right)\right] \\
\geq \sum_{t_{-i}} q\left(t_{N}\right) \sum_{S \supseteq\{i\}} \sum_{y^{S}} \mu\left(S, y^{S},\left(t_{-i}, t_{i}^{\prime}\right)\right)\left[u_{i}\left(y_{i}^{S}\left(t_{-i}, t_{i}^{\prime}\right), t_{N}\right)-u_{i}\left(x_{i}\left(t_{N}\right), t_{N}\right)\right] \\
\text { for all } i \in N \text { and } t_{i}, t_{i}^{\prime} \in T_{i} .
\end{gathered}
$$

The following are the definitions of the core that will concern us here:

An incentive compatible allocation $x \in \mathcal{A}_{N}^{*}$ is in the credible core (Dutta and Vohra (2005)) if there does not exist a random blocking plan $\mu$ against $x$ such that $\mu\left(S, y^{S}, t_{N}\right)>0$ only for one coalition $S$ and one deterministic rule $y^{S} \in \mathcal{A}_{S}$, and for $t_{N} \in \prod_{i \in S} E_{i} \times T_{-S}$, where $E_{i}$ is the set of types of agent $i$ in the support of $\mu$. Note how, in particular, the definition of incentive compatibility over an event $E$ for an $S$-allocation for a fixed coalition $S$ applies to these blocking plans, as defined above.

Thus, in a credible objection to an allocation, a fixed coalition $S$ identifies an information event over which the objection takes place. All the types consistent with that event prefer the objection to the status quo allocation after Bayesian updating of their beliefs given the event. Furthermore, no type within the relevant event wants to misrepresent its information. Finally, the types not consistent with the event do not wish to participate in the objection by pretending they are one of the types in it: to see this, in the second condition of a random blocking plan, suppose $t_{i}$ is one of these excluded types and $t_{i}^{\prime}$ one of the types in the support of $\mu$. The latter is what is called the credibility restriction in this objection. Hence the name credible objection.

If in a credible objection one drops the credibility restriction, one constructs a fine objection. The fine core is the set of allocations $x \in \mathcal{A}_{N}$ immune to fine objections (Wilson (1978)).

An incentive compatible allocation $x \in \mathcal{A}_{N}^{*}$ is in the virtual utility core (Myerson (2007)) if there does not exist a random blocking plan $\mu$ against $x$ that is measurable. 
Thus, in these objections, an agent is invited to the blocking plan, but he is not told which specific coalition is being formed. Each agent in the support of $\mu$ gets a phone call inviting him to participate in the blocking plan, and those "phone calls" are made according to $\mu$. On the basis of $\mu$, contingent on the "phone call," each agent updates his interim beliefs using Bayes' rule. Again, given this, each agent who gets the call wishes to go along with it instead of remaining at the status quo, and the appropriate incentive compatibility constraints are also imposed given $\mu$. The measurability of the plan implies that a given coalition $S$ cannot be called with different probabilities in two states $\left(t_{S}, t_{-S}^{\prime}\right)$ and $\left(t_{S}, t_{-S}^{\prime \prime}\right)$ that the coalition cannot discern.

An incentive compatible allocation $x \in \mathcal{A}_{N}^{*}$ is in the randomized mediated core (Serrano and Vohra (2007)) if there does not exist a random blocking plan $\mu$ against $x$.

For these objections, the same story applies, except that the non-measurability of $\mu$ allows for information transmission across coalitions within the blocking plan; see Section 4 for details.

Observation: The randomized mediated core is a subset of the virtual utility core, itself a subset of the credible core. Also, an incentive compatible allocation that is in the fine core is in the credible core.

Under the assumptions made so far, and if one allows average feasibility in the nummeraire (while exact feasibility is required for the other commodities), Myerson (2007) establishes the non-emptiness of the virtual utility core. Furthermore, his existence argument does not use at all the measurability of blocking plans. Therefore, it follows that, under exactly the same assumptions, the randomized mediated core is also non-empty. An alternative approach to non-emptiness is provided in Dutta and Vohra (2005). In quasilinear economies, assuming that there exists an incentive compatible ex-post core allocation, they show that the credible core is non-empty. It is easy to complete their argument to show that the randomized mediated core is also non-empty (see the proof of our Proposition 2).

To further appreciate the information transmission that goes on within each blocking plan, the reader is referred to Serrano and Vohra (2007). In that paper, each blocking plan is set up as a voting game among the players, who have to report their private information and choose whether they vote for the blocking plan or stay with the status quo. In the equilibria of these voting games, each agent performs the correct updating of his interim beliefs, using Bayes' rule and the equilibrium actions of the other types in the game. 


\subsection{Replica Economies}

The current paper is concerned with core convergence. Thus, we turn to define the replicas of the basic economies and allocations. We shall use the independent replicas introduced in Gul and Postlewaite (1992), also used in Forges, Heifetz and Minelli (2001). Each agent's utility depends only on the information contained in his replica. Because replicas are independent, the set of states in the replicated economy is the product of the sets of states for each replica.

Formally, given an economy $\mathcal{E}=\left\langle\left(u_{i}, X_{i}, \omega_{i}, T_{i}\right)_{i \in N}, q\right\rangle$, and an allocation $x \in \mathcal{A}_{N}$, replicas of $\mathcal{E}$ and $x$ are defined as follows. For every positive integer $m$, let $I_{m}=\{1,2, \ldots, m\}$. The $m$-th replica of $\mathcal{E}$ is the economy $\mathcal{E}^{m}=$ $\left\langle\left(u_{(i, j)}, X_{(i, j)}, \omega_{(i, j)}, T_{(i, j)}\right)_{(i, j) \in N \times I_{m}}, q_{m}\right\rangle$, where for all $(i, j) \in N \times I_{m}, X_{(i, j)}=$ $X_{i}, T_{(i, j)}=T_{i}$ over $\prod_{k \neq i} T_{(k, j)}, \omega_{(i, j)}=\omega_{i}, u_{(i, j)}=u_{i}: X_{i} \times \prod_{i \in N} T_{(i, j)} \mapsto R$, and

$$
q_{m}\left(t_{(1,1)}, \ldots, t_{(n, 1)}, \ldots, t_{(1, m)}, \ldots, t_{(n, m)}\right)=\prod_{j \in I_{m}} q\left(t_{(1, j)}, \ldots, t_{(n, j)}\right) .
$$

The $m$-th replica of $x$ is denoted $x^{m}$ where $x_{(i, j)}^{m}=x_{i}$ for all $(i, j) \in N \times I_{m} \cdot{ }^{4}$ Note that the set of information states changes with replication. This is different from the replication process of Serrano, Vohra and Volij (2001), which results in information becoming non-exclusive already in the second replica. This in turn makes incentive constraints redundant.

\subsection{Market Equilibrium}

The competitive market equilibrium concept that we shall employ in this paper is the ex-post Walrasian equilibrium, and we shall assume that there exists an ex-post Walrasian equilibrium allocation that is incentive compatible. Under this assumption, our negative convergence result extends also to any price-taking equilibrium concept that satisfies Property P, as first suggested in Serrano, Vohra and Volij (2001):

A price-taking equilibrium concept is said to obey Property $P$ if, whenever it is non-empty, in an economy that includes some fully informed agents, each of them receives in equilibrium a bundle that maximizes his ex-post utility over his ex-post budget constraint.

\footnotetext{
${ }^{4}$ We shall sometimes find it convenient to refer to consumer $(i, j) \in N \times I_{m}$ as consumer $i j$.
} 


\section{The Non-Convergence Result}

In the current section we show that, even if one restricts attention to replicas of allocations, i.e., even if one assumes equal treatment of agents across replicas, the virtual utility core does not converge to price-taking equilibrium allocations, no matter how many times the economy is replicated. Later in this section we shall argue that the core of any replicated economy may in addition contain allocations that violate equal treatment.

\subsection{Equal Treatment Allocations}

Our first result follows:

Proposition 1 There exists an allocation $\tilde{x}$ in an admissible economy $\mathcal{E}$ satisfying that for every $m, \tilde{x}^{m}$ is in the virtual utility core of the replicated economy $\mathcal{E}^{m}$ and that cannot be supported by any price-taking equilibrium notion that obeys Property $P$.

Proof: Consider the following economy $\mathcal{E}$. There are two consumers and two commodities. Suppose $T_{1}=\{s, t\}$ while agent 2 is uninformed (and therefore has only one type). The information state can then be described by $s$ or $t$. Suppose $s$ and $t$ are equally probable. Let $\omega_{1}=\omega_{2}=(1.5,1)$. The utility functions are as follows:

$$
\begin{array}{ll}
u_{1}\left(x^{1}, x^{2}, s\right)=\ln x^{1}+x^{2}, & u_{2}\left(x^{1}, x^{2}, s\right)=2 \ln x^{1}+x^{2} \\
u_{1}\left(x^{1}, x^{2}, t\right)=2 \ln x^{1}+x^{2}, & u_{2}\left(x^{1}, x^{2}, t\right)=\ln x^{1}+x^{2} .
\end{array}
$$

(Recall that throughout we use superscripts to index commodities and subscripts to index consumers.) Thus, the two individuals are ex ante identical, but the realized type of individual 1 determines ex post which of the two has a higher utility from consumption of the first good. ${ }^{5}$

We consider first the allocation $\hat{x}$ defined by:

$$
\begin{array}{ll}
\hat{x_{1}}(s)=(1,1.5), & \hat{x_{2}}(s)=(2,0.5) ; \\
\hat{x_{1}}(t)=(2,0.5), & \hat{x_{2}}(t)=(1,1.5) .
\end{array}
$$

\footnotetext{
${ }^{5}$ This example was first proposed in Kreps (1977) to illustrate how the set of rational expectations equilibria might be empty. It was also used in Forges, Heifetz and Minelli (2001) to show that the equal treatment property does not hold for the ex ante incentive compatible core in the two-fold replicated economy.
} 
Then each agent's ex post utility is:

$$
\begin{array}{ll}
u_{1}\left(\hat{x_{1}}(s), s\right)=1.5, & u_{2}\left(\hat{x_{2}}(s), s\right)=2 \ln 2+0.5 \\
u_{1}\left(\hat{x_{1}}(t), t\right)=2 \ln 2+0.5, & u_{2}\left(\hat{x_{2}}(t), t\right)=1.5
\end{array}
$$

Note how $\hat{x}$ must be the only allocation prescribed by an equilibrium concept that obeys Property $\mathrm{P}$ in this economy. Note also how many equilibrium concepts will obey this property here, including the ex-post Walrasian equilibrium, the constrained market equilibrium found in Wilson (1978), or the rational expectations equilibrium (which here yields the empty set). Finally, observe that $\hat{x}$ is incentive compatible.

To construct the allocation we are interested in, we modify $\hat{x}$ by requiring an additional transfer of 0.05 units of good 2 from individual 2 to individual 1 in each state. We denote the resulting allocation as $\tilde{x}$ :

$$
\begin{array}{ll}
\tilde{x_{1}}(s)=(1,1.55), & \tilde{x_{2}}(s)=(2,0.45) ; \\
\tilde{x_{1}}(t)=(2,0.55), & \tilde{x_{2}}(t)=(1,1.45) .
\end{array}
$$

This allocation yields utility levels

$$
\begin{array}{ll}
u_{1}\left(\tilde{x_{1}}(s), s\right)=1.55, & u_{2}\left(\tilde{x_{2}}(s), s\right)=2 \ln 2+0.45 \\
u_{1}\left(\tilde{x_{1}}(t), t\right)=2 \ln 2+0.55, & u_{2}\left(\tilde{x_{2}}(t), t\right)=1.45
\end{array}
$$

We consider now the independent replication process described above. Recall that we denote individual $i$ in the $j$-th replica as $(i, j)$. We present our argument in two claims.

Claim 1.1: For all $m$, both the $m$-th replicated allocations $\hat{x}^{m}$ and $\tilde{x}^{m}$ are in the credible core of $\mathcal{E}^{m}$.

Proof of Claim 1.1: We will show that the $m$-th replication of $\tilde{x}$ is in the credible core of the replicated economy $\mathcal{E}^{m}$. (The argument for $\hat{x}^{m}$ is similar and we omit it.)

Since we can check that $\tilde{x}^{m}$ is incentive compatible (each informed agent in each replica does not wish to pretend that he is of the other informed type), it suffices to show that $\tilde{x}^{m}$ is in the fine core of $\mathcal{E}^{m}$; see Dutta and Vohra (2005).

It is easy to show that $\tilde{x}$ is maximizing the sum of ex-post utilities for each state and it is ex-post individually rational. Hence, $\tilde{x}$ is in the fine core of the original economy $\mathcal{E}$. Thus, for $j=1,2, \ldots, m$, if the allocation is blocked 
by a coalition $S$ that includes both $(1, j)$ and $(2, j)$, then the allocation is also blocked by $S \backslash\{(1, j),(2, j)\}$. This implies that it is sufficient to check whether coalition $S=\{(1,1), \ldots,(1, k),(2, k+1), \ldots,(2, m)\}$ blocks the allocation $\tilde{x}^{m}$ for each $m$ and $k(0 \leq k \leq m)$. For this possible blocking coalition, we can restrict attention to information events of the form $E=$ $\prod_{j=1}^{k}\left\{r_{(1, j)}\right\} \times \prod_{j=k+1}^{m} T_{(1, j)}$, where $r_{(1, j)}=s$ or $t(1 \leq j \leq k)$.

Without loss of generality, we may assume that

$$
r_{(1, j)}= \begin{cases}s & \left(1 \leq j \leq j^{\prime}\right) \\ t & \left(j^{\prime}+1 \leq j \leq k\right)\end{cases}
$$

for some $j^{\prime}\left(0 \leq j^{\prime} \leq k\right)$. Here, $j^{\prime}=0\left(j^{\prime}=k\right)$ means that $r_{(1, j)}=t(s)$ for every $(1, j)(1 \leq j \leq k)$, respectively.

Note that any feasible allocation for coalition $S$ has to be constant on $E$, i.e., each uninformed agent must receive the same bundle in both relevant states. It follows from the quasilinearity that the optimal allocation of good 1 can be obtained as the solution to

$$
\begin{gathered}
\max \sum_{j=1}^{j^{\prime}} \ln x_{(1, j)}^{1}+\sum_{j=j^{\prime}+1}^{k} 2 \ln x_{(1, j)}^{1}+\sum_{j=k+1}^{m} \frac{1}{2}\left(2 \ln x_{(2, j)}^{1}+\ln x_{(2, j)}^{1}\right) \\
\text { s.t. } \sum_{j=1}^{k} x_{(1, j)}^{1}+\sum_{j=k+1}^{m} x_{(2, j)}^{1} \leq 1.5 m .
\end{gathered}
$$

By the first-order condition, the solution of this problem is

$$
x_{(1, i)}^{1}=\left\{\begin{array}{ll}
\lambda & \left(1 \leq j \leq j^{\prime}\right) \\
2 \lambda & \left(j^{\prime}+1 \leq j \leq k\right)
\end{array}, x_{(2, j)}^{1}=1.5 \lambda(k+1 \leq j \leq m),\right.
$$

where

$$
\lambda=\frac{1.5 m}{j^{\prime}+2\left(k-j^{\prime}\right)+1.5(m-k)}=\frac{1.5 m}{1.5 m+0.5 k-j^{\prime}} .
$$

Then the sum of interim utilities is

$$
j^{\prime} \cdot \ln \lambda+\left(k-j^{\prime}\right) \cdot 2 \ln (2 \lambda)+(m-k) \cdot 1.5 \ln (1.5 \lambda)+m .
$$

On the other hand, the sum of interim utilities of the original allocation $\tilde{x}^{m}$ for the coalition is

$$
1.55 j^{\prime}+(2 \ln 2+0.55)\left(k-j^{\prime}\right)+\frac{1}{2}\{(2 \ln 2+0.45)+1.45\} \cdot(m-k) .
$$


Now it suffices to show that (2) - (1) is non-negative for all $m, k(0 \leq k \leq$ $m)$ and $j^{\prime}\left(0 \leq j^{\prime} \leq k\right)$. We denote the difference $[(2)-(1)]$ as $g\left(j^{\prime}, k, m\right)$.

For fixed $k$ and $m(0 \leq k \leq m)$, the first-order condition with respect to the variable $j^{\prime}$ is

$$
\ln (1.5 m)-\ln \left(1.5 m+0.5 k-j^{\prime}\right) .
$$

Thus the function $g$ is minimized at $j^{\prime}=0.5 k$. Then

$$
\begin{aligned}
g(0.5 k, k, m) & =1.05 k+(\ln 2+0.95)(m-k)-1.5(m-k) \ln 1.5-m \\
& =(\ln 2-1.5 \ln 1.5-0.05)(m-k)+0.05 k \geq 0
\end{aligned}
$$

(since $\ln 2-1.5 \ln 1.5-0.05>0$ and $m \geq k \geq 0$ ). Therefore, $g\left(j^{\prime}, k, m\right) \geq 0$ for every $j^{\prime}, k$ and $m\left(0 \leq k \leq m, 0 \leq j^{\prime} \leq k\right)$. Thus, the proof of Claim 1.1 is complete.

Claim 1.2: For all $m$, both the $m$-th replicated allocations $\hat{x}^{m}$ and $\tilde{x}^{m}$ are in the virtual utility core of $\mathcal{E}^{m}$.

Proof of Claim 1.2: We will show that the $m$-th replication of $\tilde{x}$ is in the virtual utility core of the replicated economy $\mathcal{E}^{m}$. (The argument for $\hat{x}^{m}$ is similar and we omit it.)

In the proof of Claim 1.1, we have argued that the $m$-th replication of $\tilde{x}$ is in the fine core. Therefore, we have shown that for all $S \subseteq N \times I_{m}$, all $t_{S} \in T_{S}$ and all $y^{S} \in \mathcal{A}_{S}$,

$$
\sum_{i \in S} \sum_{t_{-S} \in T_{-S}} q_{m}\left(t_{-S}\right) u_{i}\left(y_{i}^{S}\left(t_{N \times I_{m}}\right), t_{N}\right) \leq \sum_{i \in S} \sum_{t_{-S} \in T_{-S}} q_{m}\left(t_{-S}\right) u_{i}\left(\tilde{x}_{i}\left(t_{N \times I_{m}}\right), t_{N}\right) .
$$

(Recall that $q_{m}$ is the probability distribution over the states in the replicated economy $\mathcal{E}^{m}$ ). The earlier inequality corresponds to a fine objection in which all information within the coalition is transmitted to its members. Now, consider a random plan $\mu$ that satisfies $T^{S}$-measurability. This inequality implies that for all $S \subseteq N \times I_{m}$, all $y^{S} \in \mathcal{A}_{S}$ and all $t_{S} \in T_{S}$,

$$
\begin{aligned}
& \sum_{i \in S} \mu\left(S, y^{S}, t_{N \times I_{m}}\right) \sum_{t_{-S} \in T_{-S}} q_{m}\left(t_{-S}\right) u_{i}\left(y_{i}^{S}\left(t_{N \times I_{m}}\right), t_{N}\right) \\
& \quad \leq \sum_{i \in S} \mu\left(S, y^{S}, t_{N \times I_{m}}\right) \sum_{t_{-S} \in T_{-S}} q_{m}\left(t_{-S}\right) u_{i}\left(\tilde{x}_{i}\left(t_{N \times I_{m}}\right), t_{N}\right) .
\end{aligned}
$$


Thus,

$$
\begin{aligned}
& \sum_{t_{S} \in T_{S}} q_{m}\left(t_{S}\right) \sum_{i \in S} \mu\left(S, y^{S}, t_{N \times I_{m}}\right) \sum_{t_{-S} \in T_{-S}} q_{m}\left(t_{-S}\right) u_{i}\left(y_{i}^{S}\left(t_{N \times I_{m}}\right), t_{N}\right) \\
& \leq \sum_{t_{S} \in T_{S}} q_{m}\left(t_{S}\right) \sum_{i \in S} \mu\left(S, y^{S}, t_{N \times I_{m}}\right) \sum_{t_{-S} \in T_{-S}} q_{m}\left(t_{-S}\right) u_{i}\left(\tilde{x}_{i}\left(t_{N}\right), t_{N}\right)
\end{aligned}
$$

which implies that

$$
\begin{aligned}
& \sum_{S \subseteq N \times I_{m}} \sum_{y^{S} \in \mathcal{A}_{S}} \sum_{t_{N \times I_{m}} \in T_{N \times I_{m}}} q_{m}\left(t_{N \times I_{m}}\right) \sum_{i \in S} \mu\left(S, y^{S}, t_{N \times I_{m}}\right) u_{i}\left(y_{i}^{S}\left(t_{N \times I_{m}}\right), t_{N}\right) \\
& \leq \sum_{S \subseteq N \times I_{m}} \sum_{y^{S} \in \mathcal{A}_{\mathcal{S}}} \sum_{t_{N \times I_{m}} \in T_{N \times I_{m}}} q_{m}\left(t_{N \times I_{m}}\right) \sum_{i \in S} \mu\left(S, y^{S}, t_{N \times I_{m}}\right) u_{i}\left(\tilde{x}_{i}\left(t_{N}\right), t_{N}\right) .
\end{aligned}
$$

If a blocking plan $\mu$ existed, we would obtain (recall equation $\left({ }^{*}\right)$ ):

$$
\begin{aligned}
& \sum_{i \in N \times I_{m}} \sum_{t_{N \times I_{m}}} q_{m}\left(t_{N \times I_{m}}\right) \sum_{S \supseteq\{i\}} \sum_{y^{S}} \mu\left(S, y^{S}, t_{N \times I_{m}}\right)\left[u_{i}\left(y_{i}^{S}\left(t_{N \times I_{m}}\right), t_{N}\right)\right. \\
& \left.=\sum_{S \subseteq N \times I_{m}} \sum_{y^{S}} \sum_{t_{N \times I_{m}}} q_{m}\left(t_{N \times I_{m}}\right) \sum_{i \in S} \mu\left(\tilde{x}_{i}\left(t_{N}\right), t_{N}\right)\right] \\
& >0,
\end{aligned}
$$

which contradicts the inequality in the previous paragraph.

Claims 1.1 and 1.2 complete the proof of Proposition 1.

\subsection{Non-Equal Treatment Allocations}

In this subsection we demonstrate that the virtual utility core of replicated economies also contains allocations that violate equal treatment across replicas, even if the economy is replicated an arbitrary number of times.

Indeed, in the same economy used in the proof of Proposition 1, consider the allocation $(\hat{x}, \ldots, \hat{x}, \tilde{x})$. We shall show that this allocation belongs to the virtual utility core of the $(m+1)$-fold replicated economy $\mathcal{E}^{m+1}$ for all $m \geq 1$. That is, in the first $m$ replicas, the bundles in $\hat{x}$ are allocated, whereas those in $\tilde{x}$ are assigned in the last replica, thereby violating the equal treatment property of this core.

We follow analogous steps to those in Claim 1.1, and first show that this allocation is in the credible core of the $(m+1)$-replicated economy. Since 
we can easily check that the proposed allocation is incentive compatible, it suffices to show that it is in the fine core.

Now we make the following observations:

Observation 1. For each state, both $\hat{x}$ and $\tilde{x}$ are maximizing the sum of ex post utilities. Both allocations are in the fine core of the original economy. Observation 2. For $k=1,2, \ldots, m, m+1$, if the allocation is blocked by a coalition $S$ that includes both $(1, k)$ and $(2, k)$, then the allocation is also blocked by $S \backslash\{(1, k),(2, k)\}$.

Observation 3. For $k=1,2, \ldots, m$, if the allocation is blocked by a coalition that includes $(2, k)$ but does not include $(1, k)$, then the allocation is also blocked by the coalition that includes $(1, k)$ but does not include $(2, k)$ (by symmetry of allocation $\hat{x}$ ).

From these observations, we can say that it is sufficient to check whether coalition $S$ is one of two possibilities: indeed, either $S=\{(1,1), \ldots,(1, m)$, $(2, m+1)\}$ or $S=\{(1,1), \ldots,(1, m),(1, m+1)\}$ block the proposed allocation for each $m$.

For the first possible blocking coalition, we can confine our attention to events of the form $E=\prod_{i \in S}\left\{r_{i}\right\} \times T_{-S}$, where $r_{i}=s$ or $t(i \in S \backslash\{(2, m+1)\})$.

Without loss of generality, we may assume that

$$
r_{(1, i)}= \begin{cases}s & (1 \leq i \leq j) \\ t & (j+1 \leq i \leq m)\end{cases}
$$

for some $j(0 \leq j \leq m)$. Here, $j=0(j=m)$ means that the types of all individuals in $S \backslash\{(2, m+1)\}$ are $s(t)$, respectively.

Note that any feasible allocation has to be constant on $E$ and utility of every individual other than $(2, m+1)$ is determined on $E$. It follows from the quasilinearity that the optimal allocation of good 1 can be obtained as the solution to

$$
\begin{gathered}
\max \sum_{i=1}^{j} \ln x_{(1, i)}^{1}+\sum_{i=j+1}^{m} 2 \ln x_{(1, i)}^{1}+\frac{1}{2} \ln x_{(2, m+1)}^{1}+\frac{1}{2} \cdot 2 \ln x_{(2, m+1)}^{1} \\
\text { s.t. } \sum_{i=1}^{m} x_{(1, i)}^{1}+x_{(2, m+1)}^{1} \leq 1.5(m+1) .
\end{gathered}
$$

By the first-order condition, the solution of this problem is

$$
x_{(1, i)}^{1}= \begin{cases}\lambda & (1 \leq i \leq j) \\ 2 \lambda & (j+1 \leq i \leq m)\end{cases}
$$


and $x_{(2, m+1)}^{1}=1.5 \lambda$, where

$$
\lambda=\frac{1.5(m+1)}{j+2(m-j)+1.5} .
$$

Then the sum of interim utilities is:

$$
j \cdot \ln \lambda+(m-j) \cdot 2 \ln (2 \lambda)+1.5 \ln (1.5 \lambda)+(m+1) .
$$

On the other hand, the sum of interim utilities of the original allocation is:

$$
1.5 j+(2 \ln 2+0.5)(m-j)+\frac{1}{2}\{(2 \ln 2+0.45)+1.45\} .
$$

Now it suffices to show that (4) - (3) is non-negative for all $m$ and $j(0 \leq$ $j \leq m)$. We write the difference:

$$
\begin{aligned}
& 1.5 j+(2 \ln 2+0.5)(m-j)+\frac{1}{2}\{(2 \ln 2+0.45)+1.45\} \\
& -[j \cdot \ln \lambda+(m-j) \cdot 2 \ln (2 \lambda)+1.5 \ln (1.5 \lambda)+(m+1)]
\end{aligned}
$$

Take partial derivative with respect to $j$ :

$$
\begin{aligned}
1 & -2 \ln 2-\ln \lambda+2 \ln (2 \lambda)-(2 m-j+1.5)[\partial \ln \lambda / \partial j] \\
& =1+\ln \lambda-(2 m-j+1.5)[\partial \ln \lambda / \partial j] \\
& =\ln \lambda=\ln \left[\frac{1.5 m+1.5}{2 m-j+1.5}\right],
\end{aligned}
$$

which vanishes at $j=0.5 m$, is negative for $j<0.5 m$ and positive thereafter. Therefore, it suffices to check the value of the function at $j=0.5 \mathrm{~m}$ for a fixed $m$ :

$$
\begin{aligned}
0.75 m+(2 \ln 2+0.5)(0.5 m)+ & \frac{1}{2}\{(2 \ln 2+0.45)+1.45\} \\
& -[(0.5 m) \cdot 2 \ln 2+1.5 \ln (1.5)+(m+1)] \\
= & \ln 2 m+\frac{1}{2}\{(2 \ln 2+0.45)+1.45\}-[(0.5 m) \cdot 2 \ln 2+1.5 \ln (1.5)+1] \\
= & \ln 2 m+\ln 2+0.95-[\ln 2 m+1.5 \ln (1.5)+1] \\
= & \ln 2-0.05-[1.5 \ln (1.5)]=0.0340 \ldots>0 .
\end{aligned}
$$

Since this value is independent of $m$, we have shown that for all $j$ satisfying that $0 \leq j \leq m$ for all $m \geq 1$, the difference (4) - (3) is positive, which contradicts that the coalition blocks the original allocation. 
If the blocking coalition contains agent $(1, m+1)$ instead of $(2, m+1)$, one can have two cases:

First, it is agent $(1, m+1)$ in state $s$. We write the utility difference, similar to (4) - (3), for this case:

$$
\begin{aligned}
& 1.5 j+(2 \ln 2+0.5)(m-j)+1.55 \\
& \quad-[(j+1) \cdot \ln \lambda+(m-j) \cdot 2 \ln (2 \lambda)+(m+1)]
\end{aligned}
$$

and now $\lambda=(1.5 m+1.5) /(2 m-j+1)$.

Again, we partially differentiate the aggregate utility difference with respect to $j$ :

$$
\begin{aligned}
1- & 2 \ln 2+\ln \lambda+2 \ln 2-(2 m-j+1)[\partial \ln \lambda / \partial j] \\
= & \ln \lambda=\ln \left[\frac{1.5 m+1.5}{2 m-j+1}\right],
\end{aligned}
$$

which vanishes at $j=0.5 m-0.5$, is negative if $j<0.5 m-0.5$ and positive thereafter.

We thus check the value of the aggregate utility difference at $j=0.5 m-$ 0.5 :

$$
\begin{aligned}
1.5(0.5 m-0.5)+(2 \ln 2+0.5)(0.5 m+0.5)+1.55 \\
\quad-[(0.5 m+0.5) 2 \ln 2+(m+1)] \\
=0.75 m-0.75+\ln 2(m+1)+0.25 m+0.25+1.55-[(m+1)(1+\ln 2)] \\
=0.05>0,
\end{aligned}
$$

and also independent of $m$, so we are also done with this case. Finally, we have case 2 : agent $(1, m+1)$ acts in state $t$. The utility difference similar to $(2)-(1)$ is now:

$$
\begin{aligned}
& 1.5 j+(2 \ln 2+0.5)(m-j)+2 \ln 2+0.55 \\
& \quad-\quad[j \cdot \ln \lambda+(m+1-j) \cdot 2 \ln (2 \lambda)+(m+1)],
\end{aligned}
$$

where now $\lambda=(1.5 m+1.5) /(2 m-j+2)$.

Differentiating partially with respect to $j$, one gets:

$$
\begin{aligned}
1 & -2 \ln 2-[\ln \lambda-2 \ln (2 \lambda)+(2 m-j+2)(\partial \ln \lambda / \partial j)] \\
& =-2 \ln 2-[\ln \lambda-2 \ln (2 \lambda)] \\
& =\ln \lambda=\ln \left[\frac{1.5 m+1.5}{2 m-j+2}\right]
\end{aligned}
$$


which vanishes at $j=0.5 m+0.5$, is negative at $j<0.5 m+0.5$, and positive thereafter. Therefore, it suffices to check the value of the aggregate utility difference at $j=0.5 m+0.5$ :

$$
\begin{aligned}
& 1.5(0.5 m+0.5)+(2 \ln 2+0.5)(0.5 m-0.5) \\
& +2 \ln 2+0.55-[(m+1)(\ln 2+1)] \\
& =0.05>0,
\end{aligned}
$$

also independent of $m$.

Thus, now the proof is complete. No such blocking coalition exists and the proposed allocation is in the incentive compatible fine core, and hence, also in the credible core.

The arguments to show that the allocation is in the virtual utility core are similar to the inequalities derived in Claim 1.2, and we therefore omit them.

\section{Convergence of the Randomized Mediated Core}

In this section we show that the equal treatment allocations of the randomized mediated core converge to the set of ex-post Walrasian allocations as the economy is replicated enough times. We do not know whether the assumption of equal treatment is important to obtain this result; we have not been able to either prove it or disprove it.

We begin by devoting some space to the issue of timing of actions in the random allocation cores studied in this paper (the arguments also apply to Myerson's virtual utility core, since the measurability of the blocking plans is not important for this).

\subsection{Timing in Random Blockings}

Following Myerson (2007), the discussions of a blocking plan against a status quo in a context of incomplete information take place after the truthful reports for the status quo have occurred, but before the implementation of such a status quo. Therefore, one can specify the following timing of actions within the blocking plan $\mu$ against an incentive compatible $x$ : 
- Stage 0: types are reported to sustain $x$ as an incentive compatible allocation. These type reports will be used every time the status quo needs to be implemented, and their reports are made with independence of any potential blocking plan (if this is not the case and there is complete forward-looking behavior, i.e., agents envisioning each possible blocking plan before they report their types to sustain the status quo, one would arrive at a concept far afield from the core).

- Stage 1: this and the next stages describe the timing of actions within the blocking plan $\mu$ itself. Types are reported again after players have been informed about $\mu$. Each player is only informed about the instances in which $\mu$ calls him to act. The type reports of this stage are used only if and when the blocking plan is implemented, and thus, the conditioning used in the equations of the definition of a random blocking plan (equation $(* *)$ in Section 2) is correct.

- Stage 2: phone calls are made by the blocking mediator, taking into account the probabilities $\mu\left(S, y^{S}, t_{N}\right)$, which use already the correct types reported within $\mu$. Note in particular how a non-measurable plan is perfectly possible, allowing information transmission from coalition to coalition within $\mu$. On the other hand, the plan must be measurable with respect to the information of the union of coalitions within $\mu$.

- Stage 3: each agent in the support of $\mu$ is asked to either accept or reject the blocking plan. Again, the relevant conditioning of beliefs is as in the equations that describe the random blocking plan (equation $(*)$ in Section 2).

To illustrate the concept of randomized mediated core, we shall consider again the economy in the proof of Claim 1.1. Indeed, we show now that $\tilde{x}$, which was shown to be in the virtual utility core, is not in the randomized mediated core.

Example 1 Consider the economy in the proof of Proposition 1. First, note that the second replication of $\tilde{x}$ is not in the ex-post core. For instance, it is blocked by coalition $S=\{(2,1),(1,2),(2,2)\}$ in state $(s, s)$ (this notation means that for each of the two replicas the type of individual 1 is s). We denote an ex-post blocking allocation bundle for $S$ as $y^{S}(s, s)$. 
For coalition $S^{\prime}=\{(1,1),(2,1)\}$, we define an allocation bundle $y^{S^{\prime}}(s, s)$ as follows: $y_{(1,1)}^{S^{\prime}}(s, s)=(1,1.56)$ and $y_{(2,1)}^{S^{\prime}}(s, s)=(2,0.44)$, which can be obtained from $\tilde{x}(s)$ by an additional transfer of 0.01 units of commodity 2 from individual $(2,1)$ to individual $(1,1)$.

Then, we consider the following blocking plan $\mu: \mu\left(S, y^{S},(s, s)\right)=1-\varepsilon(\varepsilon$ being a very small positive number $), \mu\left(S^{\prime}, y^{S^{\prime}},(s, s)\right)=\varepsilon$, and $\mu\left(T, y^{T}, r\right)=0$ for all coalitions $T$ and states $r \neq(s, s)$. Then, the blocking plan $\mu$ makes all individuals involved better off. (Choosing $\varepsilon$ sufficiently small, individual $(2,1)$ is better off. Because agent $(1,1)$ only participates in the blocking plan with probability $\varepsilon$, in the event he receives the phone call from the blocking mediator, he accepts the plan because he also improves. Implicitly, he believes that the status quo is still available in the event he is not part of the blocking plan, and under these beliefs, types are reported truthfully to the blocking plan. $)^{6}$ Finally, it is easy to choose $y^{S}(s, s)$ appropriately to ensure that the blocking plan satisfies incentive compatibility.

\subsection{Equilibrium Inclusion in the Core}

In this subsection we show that, in our domain of quasilinear economies, the set of ex-post Walrasian allocation rules that are incentive compatible is in the randomized mediated core.

Proposition 2 Consider a quasilinear exchange economy $\mathcal{E}$ in our admissible class. Let $x^{*}$ be an ex-post Walrasian equilibrium allocation rule satisfying incentive compatibility. Then, for all $m, x^{* m}$ is in the randomized mediated core.

Proof: By hypothesis, $x^{*}$ is incentive compatible. So we need to show that there does not exist any random blocking plan that improves upon $x^{* m}$.

Since an ex-post Walrasian allocation is an element of the ex-post core, we can say that for all $S \subseteq N \times I_{m}$, all $t_{N \times I_{m}} \in T_{N \times I_{m}}$ and $y^{S} \in \mathcal{A}_{S}$,

$$
\sum_{i \in S} u_{i}\left(y_{i}^{S}\left(t_{N \times I_{m}}\right), t_{N}\right) \leq \sum_{i \in S} u_{i}\left(x_{i}^{* m}\left(t_{N \times I_{m}}\right), t_{N}\right) .
$$

\footnotetext{
${ }^{6}$ These "infeasible beliefs" are part of equations $\left(^{*}\right)$ and $(* *)$, and concern both the virtual utility core and the randomized mediated core, which, as we are showing, have very different convergence properties. Similar infeasible beliefs also occur in the blockings used in the inner core with complete information (see, e.g., Myerson (1991, p. 462-468)).
} 
This corresponds to an ex-post objection. Now, consider a random plan $\mu$ (without measurability). This inequality implies that for all $S \subseteq N \times I_{m}$, all $y^{S} \in \mathcal{A}_{S}$ and all $t_{N \times I_{m}} \in T_{N \times I_{m}}$,

$$
\sum_{i \in S} \mu\left(S, y^{S}, t_{N \times I_{m}}\right) u_{i}\left(y_{i}^{S}\left(t_{N \times I_{m}}\right), t_{N}\right) \leq \sum_{i \in S} \mu\left(S, y^{S}, t_{N \times I_{m}}\right) u_{i}\left(x_{i}^{* m}\left(t_{N \times I_{m}}\right), t_{N}\right) .
$$

Thus,

$$
\begin{aligned}
& \sum_{S \subseteq N \times I_{m}} \sum_{y^{S} \in \mathcal{A}_{S}} \sum_{t_{N \times I_{m}} \in T_{N \times I_{m}}} q_{m}\left(t_{N \times I_{m}}\right) \sum_{i \in S} \mu\left(S, y^{S}, t_{N \times I_{m}}\right) u_{i}\left(y_{i}^{S}\left(t_{N \times I_{m}}\right), t_{N}\right) \\
& \leq \sum_{S \subseteq N \times I_{m}} \sum_{y^{S \in \mathcal{A}_{\mathcal{S}}}} \sum_{t_{N \times I_{m}} \in T_{N \times I_{m}}} q_{m}\left(t_{N \times I_{m}}\right) \sum_{i \in S} \mu\left(S, y^{S}, t_{N \times I_{m}}\right) u_{i}\left(x_{i}^{* m}\left(t_{N}\right), t_{N}\right) .
\end{aligned}
$$

If a blocking plan $\mu$ existed, we would obtain (recall equation $\left({ }^{*}\right)$ ):

$$
\begin{aligned}
& \sum_{i \in N \times I_{m}} \sum_{t_{N \times I_{m}}} q_{m}\left(t_{N \times I_{m}}\right) \sum_{S \supseteq\{i\}} \sum_{y^{S}} \mu\left(S, y^{S}, t_{N \times I_{m}}\right)\left[u_{i}\left(y_{i}^{S}\left(t_{N \times I_{m}}\right), t_{N}\right)\right. \\
& \left.=\sum_{S \subseteq N \times I_{m}} \sum_{y^{S}} \sum_{t_{N \times I_{m}}} q_{m}\left(t_{N \times I_{m}}\right) \sum_{i \in S} \mu\left(S, y_{i}^{* m}\left(t_{N}\right), t_{N}\right)\right] \\
& >0, \\
& \left.-t_{N \times I_{m}}\right)\left[u_{i}\left(y_{i}^{S}\left(t_{N \times I_{m}}\right), t_{N}\right)\right. \\
& \left.\left.-x_{i}^{* m}\left(t_{N}\right), t_{N}\right)\right]
\end{aligned}
$$

which contradicts the inequality in the previous paragraph.

Remark: Note the parallel steps followed by the proofs of Claim 1.2 and of Proposition 2. There is an important difference, though. While the allocation $\tilde{x}^{m}$ of the proof of Claim 1.2 is in the fine core of every replica, $x^{* m}$ is in the ex-post core of every replica. This difference matters: in the former case, the non-existence of a fine objection leads, through the use of measurable blocking plans, to the non-existence of an objection in the sense of the virtual utility core. In the case of the latter, not having ex-post objections in any state leads, through the use of (measurable or not) blocking plans, to the non-existence of an objection in the sense of the randomized mediated core.

\subsection{Convergence}

Next, we show that the equal-treatment strictly incentive compatible allocations in the randomized mediated core converge, as the economy is replicated 
enough times, to the set of incentive compatible ex-post Walrasian allocations.

Proposition 3 Suppose that, in every ex-post state, the assumptions to obtain the core convergence theorem of Debreu and Scarf (1963) are satisfied. Then, if $x$ is a strictly incentive compatible allocation rule in the economy $\mathcal{E}$ and its replica $x^{m}$ is in the randomized mediated core of $\mathcal{E}^{m}$ for every $m, x$ must be an ex-post Walrasian allocation rule.

Proof: Suppose not. That is, let $x$ be a strictly incentive compatible allocation rule whose replica is in the randomized mediated core of every replicated economy, but suppose that $x\left(t_{N}\right)$ is not a Walrasian allocation for some $t_{N}$. We shall show that there exists a random blocking plan that improves upon $x$ for some replica of the original economy.

By Debreu and Scarf (1963), there exists $m$ such that in the $m$-th replication of the ex-post economy in $t_{N}$, there exists a coalition $S$ and a feasible plan $y^{S} \in \mathcal{A}_{S}, S \subseteq N \times I_{m}$, such that $u_{i}\left(y_{i}^{S}\left(\bar{t}_{m}\right), t_{N}\right)>u_{i}\left(x_{i}\left(t_{N}\right), t_{N}\right)$ for all $i \in S$, where $\bar{t}_{m}:=\left(t_{N}, t_{N}, \ldots, t_{N}\right)$. Consider another allocation rule $x^{\prime}$ arbitrarily close to $x$, also strictly incentive compatible, satisfying that $x_{i}=x_{i}^{\prime}$ for all $i \notin S$.

For this $m$, let us consider the following blocking plan $\mu$ for $N \times I_{m}$ : $\mu\left(S, y^{S}\left(\bar{t}_{m}\right), \bar{t}_{m}\right)=\varepsilon>0, \mu\left(N \times I_{m},\left(x^{\prime}\left(t_{N}\right)\right)^{m}, \bar{t}_{m}\right)=1-\varepsilon>0$ (where $\left(x^{\prime}\left(t_{N}\right)\right)^{m}$ represents the $m$-th replication of $\left.x^{\prime}\left(t_{N}\right)\right)$ and $\mu$ assigns zero for any other state.

Since $x^{\prime}$ satisfies incentive compatibility, we can see that both conditions $(*)$ and $(* *)$ of Section 2 are satisfied for any $i$ not in $S$. For $i \in S$, conditions $(*)$ are clearly satisfied. For the conditions $(* *)$-incentive compatibility-, one has two cases:

Case 1: Agent $i$ 's true type is $t_{i}$ and he reports $t_{i}^{\prime}$. Then, the LHS of $(* *)$ is, because of the state probability, approximately proportional to $\varepsilon\left[u_{i}\left(y_{i}^{S}\left(\bar{t}_{m}\right), t_{N}\right)-u_{i}\left(x\left(t_{N}\right), t_{N}\right)\right]>0$, while the RHS is 0.

Case 2: Agent $i$ 's true type is $t_{i}^{\prime}$ and he reports $t_{i}$. Let $t^{\prime}:=\left(t_{i}^{\prime}, t_{N \backslash\{i\}}\right)$. Then, the LHS of $\left(^{* *}\right)$ is 0 , whereas the RHS is proportional to $\varepsilon\left[u_{i}\left(y_{i}^{S}\left(\bar{t}_{m}\right), t^{\prime}\right)\right.$ $\left.-u_{i}\left(x\left(t^{\prime}\right), t^{\prime}\right)\right]+(1-\varepsilon)\left[u_{i}\left(x_{i}^{\prime}\left(t_{N}\right), t^{\prime}\right)-u_{i}\left(x_{i}\left(t^{\prime}\right), t^{\prime}\right)\right]$.

Since $u_{i}\left(x_{i}^{\prime}\left(t_{N}\right), t^{\prime}\right)-u_{i}\left(x_{i}\left(t^{\prime}\right), t^{\prime}\right)<0$ by strict incentive compatibility, by taking $\varepsilon$ small enough, we can obtain that the RHS is negative. 
Remark: The proof of Proposition 3 does not use the assumption that the set of incentive compatible ex-post Walrasian allocations is non-empty. In its absence, the proposition implies the non-existence of equal-treatment allocations in the randomized mediated core of sufficiently large replicas.

\section{Concluding Remarks}

This paper has offered a variety of convergence results for different core notions in quasilinear economies. The main factor that accounts for the difference in results is the amount of information transmission that one permits each coalition to use. If random coalition formation is possible and information can be used, within a blocking plan, from coalition to coalition in the plan, a positive convergence result was obtained to the set of incentive compatible ex-post Walrasian allocations, whenever this is non-empty.

We finally observe that, while we have been using the incentive constraints in our analysis -in part, because so do the cores that concerned us here-, all our results extend to the case in which such constraints are not imposed. In particular, the economy described in the proof of Proposition 1 still shows that the proposed allocation remains in the core of every replica. That is, even if non-incentive compatible random blocking plans are possible and coalitions are allowed to use random allocations, no such blocking exists for $\tilde{x}$. Also, the proofs of Propositions 2 and 3 still apply if one does not make use of incentive compatibility constraints, to show that the corresponding version of the randomized mediated core converges to the set of ex-post Walrasian allocations. ${ }^{7}$

\footnotetext{
${ }^{7}$ Ignoring incentive constraints, Dutta and Vohra (2005) show that in quasilinear economies the ex-post core is included in the fine core, which is therefore non-empty. Their argument can be extended in a straightforward way -as in the proof of our Proposition 2- to show that the corresponding version of the randomized mediated core is also non-empty in this case. See also de Clippel (2007) for a different core convergence result when incentive constraints are ignored.
} 


\section{References}

Anderson, R. M. (2008). "Core Convergence," In S. Durlauff and L. Blume (eds.): The New Palgrave Dictionary of Economics (2nd edition), McMillan, London, forthcoming.

Aumann, R. J., (1964). "Markets with a Continuum of Traders," Econometrica 32, 39-50.

Clippel, G. de, (2007). "The Type-Agent Core of Exchange Economies with Asymmetric Information," Journal of Economic Theory 135, 144-158.

Clippel, G. de, Minelli, E., (2005). "Two Remarks on the Inner Core," Games and Economic Behavior 50, 143-154.

Debreu, G., Scarf, H., (1963). "A Limit Theorem on the Core of an Economy," International Economic Review 4, 235-246.

Dutta, B., Vohra, R., (2005). "Incomplete Information, Credibility and the Core," Mathematical Social Sciences 50, 148-165.

Einy, E., Moreno, D., Shitovitz, B., (2000). "Rational Expectations Equilibria and the Ex-Post Core of an Economy with Asymmetric Information," Journal of Mathematical Economics 34, 527-535.

Forges, F., Heifetz, A., Minelli, E., (2001). "Incentive Compatible Core and Competitive Equilibria in Differential Information Economies", Economic Theory 18, 349-365.

Forges, F., Mertens, J.-F., Vohra, R., (2002). "The Ex Ante Incentive Compatible Core in the Absence of Wealth Effects," Econometrica 70, 18651892.

Forges, F., Minelli, E., Vohra, R., (2002). "Incentives and the Core of an Exchange Economy: a Survey," Journal of Mathematical Economics 38, $1-41$.

Gul, F., Postlewaite, A., (1992). "Asymptotic Efficiency in Large Exchange Economies with Asymmetric Information," Econometrica 60, 1273-1292.

Kreps, D., (1977). "A Note on Fulfilled Expectations Equilibria," Journal of Economic Theory 14, 32-43.

Myerson, R. B., (1991). Game Theory: Analysis of Conflict, Harvard University Press, Cambridge, MA.

Myerson, R. B., (2007). "Virtual Utility and the Core for Games with Incomplete Information," Journal of Economic Theory 136, 260-285.

Postlewaite, A., Schmeidler D., (1986). "Implementation in Differential Information Economies," Journal of Economic Theory 39, 14-33. 
Qin, C.-Z., (1993). "The Inner Core and the Strictly Inhibitive Set," Journal of Economic Theory 59, 431-444.

Serrano, R., Vohra, R., (2007). "Information Transmission in Coalitional Voting Games," Journal of Economic Theory 134, 117-137.

Serrano, R., Vohra, R., Volij, O., (2001). "On the Failure of Core Convergence in Economies with Asymmetric Information," Econometrica 69, $1685-1696$.

Vohra, R., (1999). "Incomplete Information, Incentive Compatibility and the Core," Journal of Economic Theory 86, 123-147.

Wilson, R., (1978). "Information, Efficiency and the Core of an Economy," Econometrica 46, 807-816. 